\title{
Deep neural networks and visuo-semantic models explain complementary components of human ventral-stream representational dynamics
}

\author{
Kamila M Jozwik ${ }^{1}$, Tim C Kietzmann ${ }^{2}$, Radoslaw M Cichy ${ }^{3}$, Nikolaus Kriegeskorte ${ }^{4}$, and Marieke Mur ${ }^{5,6}$ \\ ${ }^{1}$ Department of Psychology, University of Cambridge, Cambridge, UK. \\ ${ }^{2}$ Donders Institute for Brain, Cognition and Behaviour, Radboud University, Nijmegen, Netherlands. \\ ${ }^{3}$ Freie Universität Berlin, Department of Education and Psychology, Berlin, Germany. \\ ${ }^{4}$ Zuckerman Mind Brain Behavior Institute, Columbia University, New York, USA. \\ ${ }^{5}$ Department of Psychology, Western University, London, Canada \\ ${ }^{6}$ Department of Computer Science, Western University, London, Canada
}

\begin{abstract}
Deep neural networks (DNNs) are promising models of the cortical computations supporting human object recognition. However, despite their ability to explain a significant portion of variance in neural data, the agreement between models and brain representational dynamics is far from perfect. Here, we address this issue by asking which representational features are currently unaccounted for in neural timeseries data, estimated for multiple areas of the human ventral stream via source-reconstructed magnetoencephalography (MEG) data. In particular, we focus on the ability of visuo-semantic models, consisting of human-generated labels of higher-level object features and categories, to explain variance beyond the explanatory power of DNNs alone. We report a gradual transition in the importance of visuo-semantic features from early to higher-level areas along the ventral stream. While early visual areas are better explained by DNN features, higher-level cortical dynamics are best accounted for by visuo-semantic models. These results suggest that current DNNs fail to fully capture the visuo-semantic features represented in higher-level human visual cortex and suggest a path towards more accurate models of ventral stream computations.
\end{abstract}

vision | object recognition | features | categories | recurrent deep neural networks | source-reconstructed MEG data

Correspondence: jozwik.kamila@gmail.com,mmur@uwo.ca

\section{Introduction}

When we view objects in our visual environment, the neural representation of these objects dynamically unfolds over time across the cortical hierarchy of the ventral visual stream. In brain recordings from both humans and nonhuman primates, this dynamic representational unfolding can be quantified from neural population activity, showing a staggered emergence of ecologically relevant object information such as face features, followed by object categories, and then the individuation of these inputs into specific exemplars (1-10). These neural reverberations are thought to reflect the cortical computations that support object recognition.

Deep neural networks (DNNs) have recently emerged as a promising computational framework for modeling these cortical computations $(11,12)$. DNNs explain significant variance in neural data obtained from visual cortex in both humans and nonhuman primates (13-18). More recent DNNs that incorporate dynamics through recurrent processing provide additional explanatory power, possibly by better approximating the dynamic computations that the brain relies on for perceptual inference $(10,19-26)$. However, even the most state-of-the-art DNNs still leave a significant amount of variance in brain representations unexplained (17), and differences among task-trained feedforward architectures are small (27, 28), even after training and fitting (18). This raises the question of what representational features are left unaccounted for in the neural data.

To address this question, we here enriched our modeling strategy with human-derived visuo-semantic object information. Visuo-semantic models consist of human-generated labels of object properties, including higher-level object features such as "eye", and categories such as "face". These object properties explain significant amounts of response variance in higher-level primate visual cortex (29-40). Moreover, visuo-semantic models outperform DNNs at predicting perceived object similarity in humans (41). We hypothesized that visuo-semantic models capture representational features in neural data that DNNs fail to account for.

We tested this hypothesis on temporally resolved magnetoencephalography (MEG) data, which can capture representational dynamics at a millisecond timescale. Human brain data acquired at this rapid sampling rate provide rich information about temporal dynamics, and by extension, about the underlying neural computations. For example, in an MEG study examining a visual delayed-match-to-sample task, time series analyses revealed distinct representational states over cue, delay, and response periods within individual experimental trials (9). In another MEG study that used source reconstruction to localize time series to distinct areas of the ventral stream, time series analyses revealed temporal inter-dependencies between areas suggestive of 
recurrent information processing (10).

In this work, we used representational similarity analysis to test both DNNs and visuo-semantic models for their ability to mirror the representational dynamics observed across multiple ventral stream areas in the human brain. By using source-reconstructed MEG data, we localized three distinct stages of processing in the ventral cortical hierarchy: lower-level, intermediate, and higher-level visual cortex (10). At each stage of processing, we tested both model classes for their ability to explain variance in the temporally evolving representations. Our modeling strategy revealed that DNNs and visuo-semantic models explain complementary components of ventral-stream representational dynamics. DNNs outperformed visuo-semantic models in lower-level visual cortex but failed to capture a significant component of representational variance in higher-level visual cortex. This variance was accounted for by visuo-semantic labels of object parts and basic categories. Our findings point towards priorities for future modeling efforts, which should move to better capturing the dynamic computations that give rise to the emergence of visuo-semantic features represented in higher-level human visual cortex.

\section{Results}

DNNs better explain lower-level visual representations, visuo-semantic models better explain higher-level visual representations. We first evaluated the overall ability of the DNN and visuo-semantic models to explain the time course of information processing along the human ventral visual stream. We hypothesized that visuo-semantic models capture representational features in neural data that DNNs may fail to account for. Figure 1 shows an overview of our approach. We analyzed MEG data acquired from human participants while they were viewing object images from a range of natural categories. We computed representational dissimilarity matrix movies (RDM movies) from the source-reconstructed MEG data to characterize how the ventral-stream object representations evolved over time. We explained variance in the RDM movies using a DNN model, consisting of internal object representations in layers of CORnet-Z and CORnet-R (22) as well as a visuo-semantic model, consisting of human-generated labels of object features and categories (40). We here include two DNN variants: CORnet-Z, a purely feedforward model, and CORnet-R, a locally recurrent variant. We computed model predictions by linearly combining either all DNN layers or all visuo-semantic labels to best explain variance in the RDM movies across time. We evaluated the model predictions on data for images left out during fitting. For each model, we tested if and when the variance explained in the RDM movies exceeded the prestimulus baseline. We also tested if and when the amounts of explained variance differed between the two models.

For lower-level visual cortex (V1-3), the DNN model explained significant amounts of variance between 60 and 638 , and 818 and $884 \mathrm{~ms}$ after stimulus onset, while the visuo-semantic model did so between 118 and $660 \mathrm{~ms}$ after stimulus onset (Figure 2a). The DNN model explained more variance than the visuo-semantic model during the early (62 - $128 \mathrm{~ms})$ as well as the late (382 - $546 \mathrm{~ms}$, on and off 812 - $996 \mathrm{~ms}$ ) phases of the response. For intermediate visual cortex (V4t/LO), the DNN model explained variance between 62 and $610 \mathrm{~ms}$ after stimulus onset, while the visuo-semantic model explained variance between 110 and $562 \mathrm{~ms}$ after stimulus onset (Figure 2a). The DNN model explained more variance than the visuo-semantic model early $(60$ - $90 \mathrm{~ms})$ but not late in time. The results for lower-level and intermediate visual cortex indicate that the DNN model outperformed the visuo-semantic model at explaining object representations, especially during the early phase of the response $(<125 \mathrm{~ms}$ after stimulus onset). In contrast, for higher-level visual cortex (IT/PHC), the visuo-semantic model outperformed the DNN model. The DNN model explained variance only between 182 and $270 \mathrm{~ms}$ after stimulus onset (Figure 2a). The visuo-semantic model explained variance during a longer time window, between 96 and $658 \mathrm{~ms}$ after stimulus onset (Figure 2a). Furthermore, the visuo-semantic model explained more variance than the DNN model between 146 and $628 \mathrm{~ms}$ after stimulus onset. In summary, the results across the ventral stream regions exhibit a reversal in which model best explains variance in the RDM movies, from the DNN model in lower-level visual cortex to the visuo-semantic model in higher-level visual cortex.

Visuo-semantic models explain unique variance in higher-level visual representations. Our results suggest that DNNs and visuo-semantic models explain complementary components of human ventral-stream representational dynamics. To explicitly test this hypothesis, we assessed the unique contributions of the two models. For this, we first computed the best RDM predictions for each model class, and then used the resulting cross-validated RDM predictions in a second-level general linear model (GLM) in which we combined the two model classes. We then computed the unique contribution of a model class by subtracting the variance explained by the reduced model (i.e. the GLM without the model class of interest) from the variance explained by the full model (including both model classes). For lower-level visual cortex (V1-3), the DNN model explained unique variance between 60 and 638, and 818 and $884 \mathrm{~ms}$ after stimulus onset, while the visuo-semantic model did so between 124 and $412 \mathrm{~ms}$ after stimulus onset (Figure 2b). For intermediate visual cortex (V4t/LO), the DNN model explained unique variance between 62 and $610 \mathrm{~ms}$ after stimulus onset, while the visuo-semantic model did so between 118 and $546 \mathrm{~ms}$ after stimulus onset (Figure 2b). These results indicate that the DNN and visuo-semantic models each explained a significant amount 
bioRxiv preprint doi: https://doi.org/10.1101/2021.10.25.465583; this version posted October 26, 2021. The copyright holder for this preprint (which was not certified by peer review) is the author/funder, who has granted bioRxiv a license to display the preprint in perpetuity. It is made available under aCC-BY-NC-ND 4.0 International license.

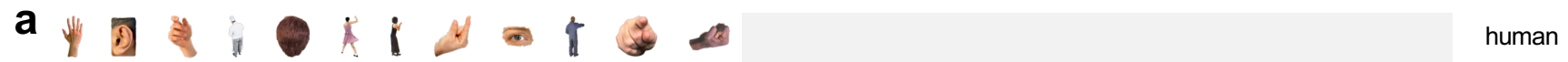

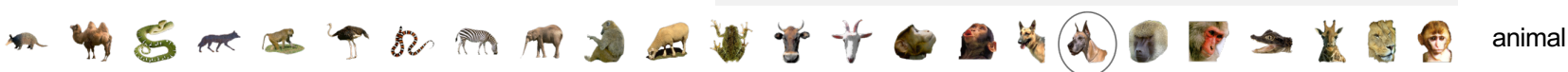

 a}

b

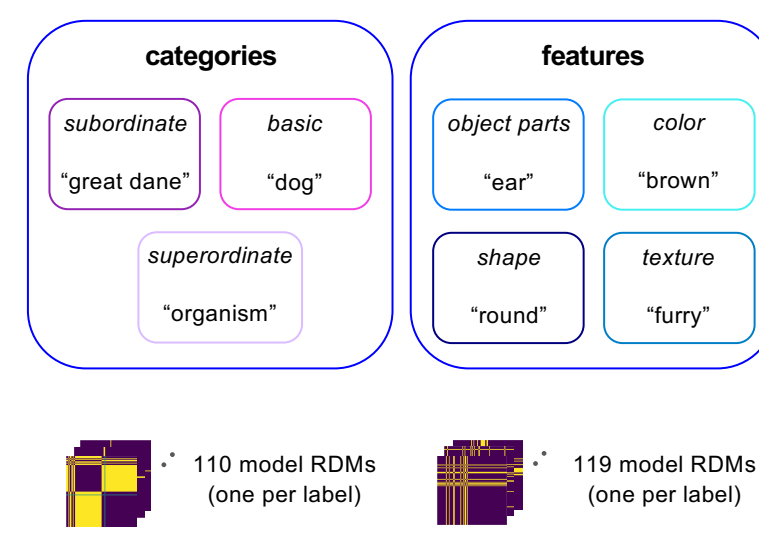

deep neural networks (DNNs)
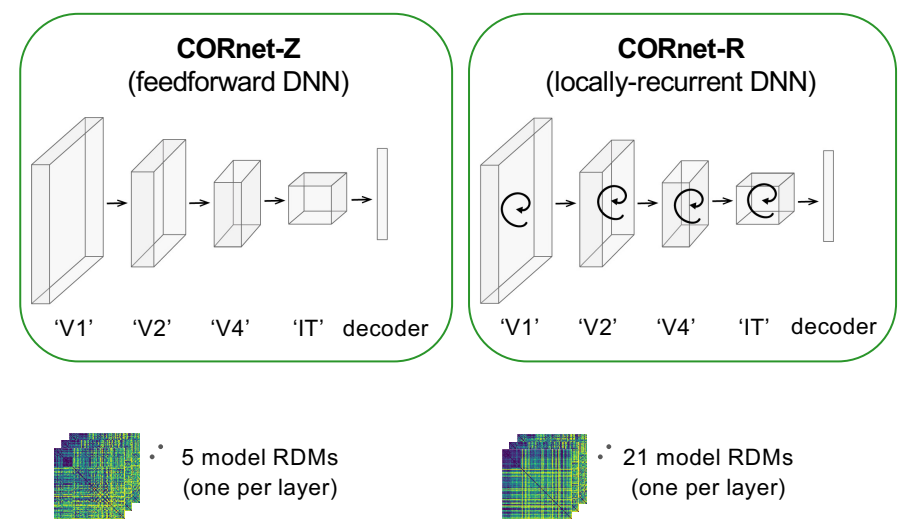

d human source-reconstructed MEG data (example participant)

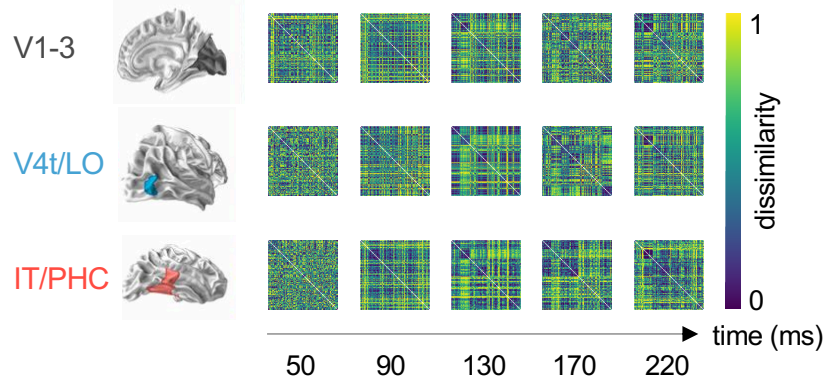

e

explain data with models (example model; fit model RDMs at each timepoint)

Fig. 1. Schematic overview of approach: stimulus set, models, data, and model fitting.

a) Stimulus set. Stimuli are 92 colored images of real-world objects spanning a range of categories, including humans, non-human animals, natural objects, and manmade objects (human face images are not shown and are replaced by gray squares according to bioRxiv's policy on posting images of individuals). b) Visuo-semantic models and deep neural networks (DNNs). Visuo-semantic models consist of human-generated labels of object features and categories for the 92 images. Example labels are shown for the dog face encircled in panel (a). DNNs are feedforward and locally recurrent CORnet architectures trained with category supervision on ILSVRC. These architectures are inspired by the processing stages of the primate ventral visual stream: from V1 to inferior temporal cortex (IT). c) Object representations for each model. We characterized object representations by computing representational dissimilarity matrices (RDMs). We computed one RDM per model dimension, i.e. one for each visuo-semantic label or DNN layer. For each visuo-semantic model dimension, RDMs were computed by extracting the value for each image on that dimension and computing pairwise dissimilarities (squared difference) between the values. For each CORnet-Z and CORnet-R layer, RDMs were computed by extracting an activity pattern across model units for each image and computing pairwise dissimilarities (1 minus Spearman's r) between the activity patterns. d) Human source-reconstructed MEG data for an example participant. MEG data were acquired in 15 healthy adult human participants while they were viewing the 92 images (stimulus duration: $500 \mathrm{~ms}$ ). We analyzed source-reconstructed data from three ROIs: V1-3, V4t/LO, and IT/PHC. We computed an RDM for each participant, region, and time point. RDMs were computed by extracting an activity pattern for each image and computing pairwise dissimilarities (1 minus Pearson's $r$ ) between the activity patterns. e) Schematic overview of model fitting procedure. We tested two model classes: a visuo-semantic model consisting of all category and feature RDMs and a DNN model consisting of all CORnet-Z and CORnet-R layer RDMs. The respective model RDMs serve as predictors. We fitted the two models to the MEG RDMs for each participant, region, and time point, using cross-validated non-negative least squares regression. 
bioRxiv preprint doi: https://doi.org/10.1101/2021.10.25.465583; this version posted October 26, 2021. The copyright holder for this preprint (which was not certified by peer review) is the author/funder, who has granted bioRxiv a license to display the preprint in perpetuity. It is made available under aCC-BY-NC-ND 4.0 International license.

a

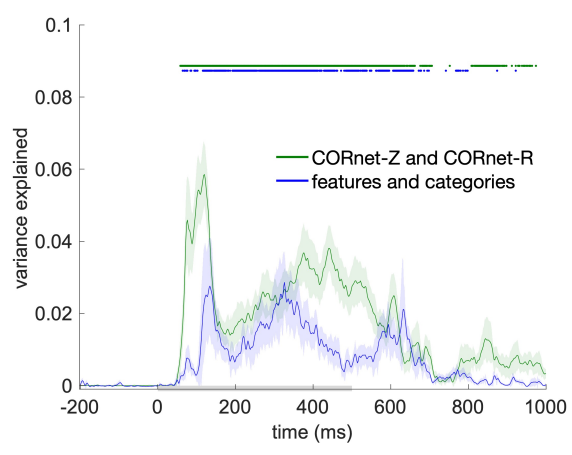

b

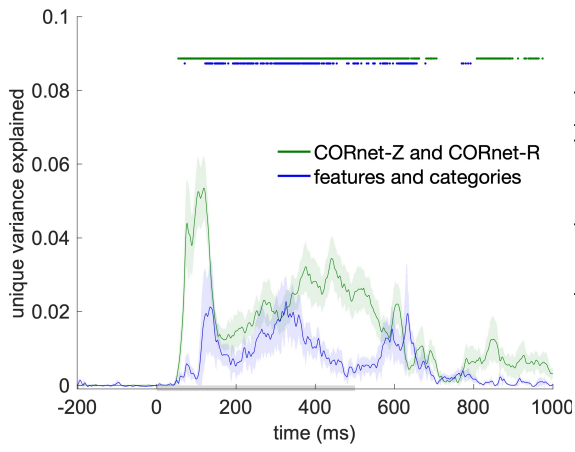

V4t/LO
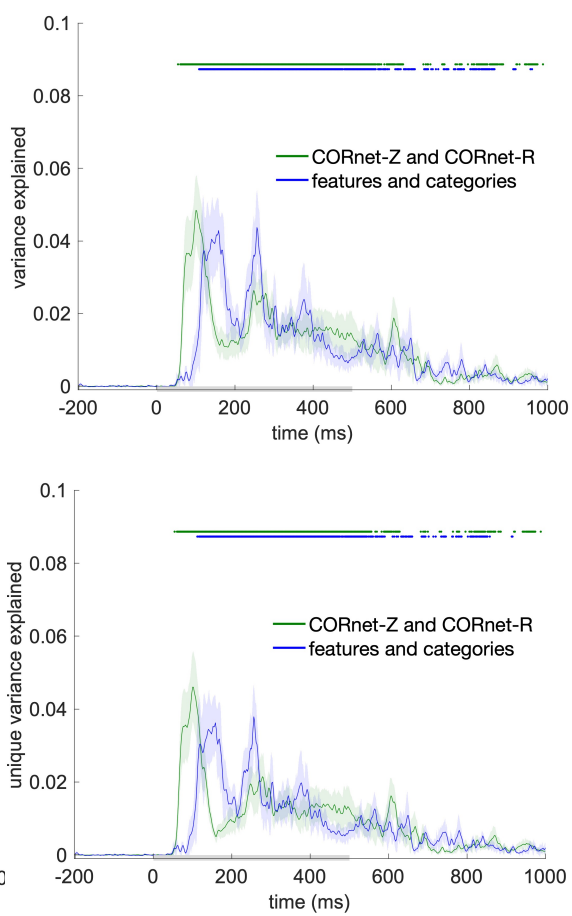

IT/PHC
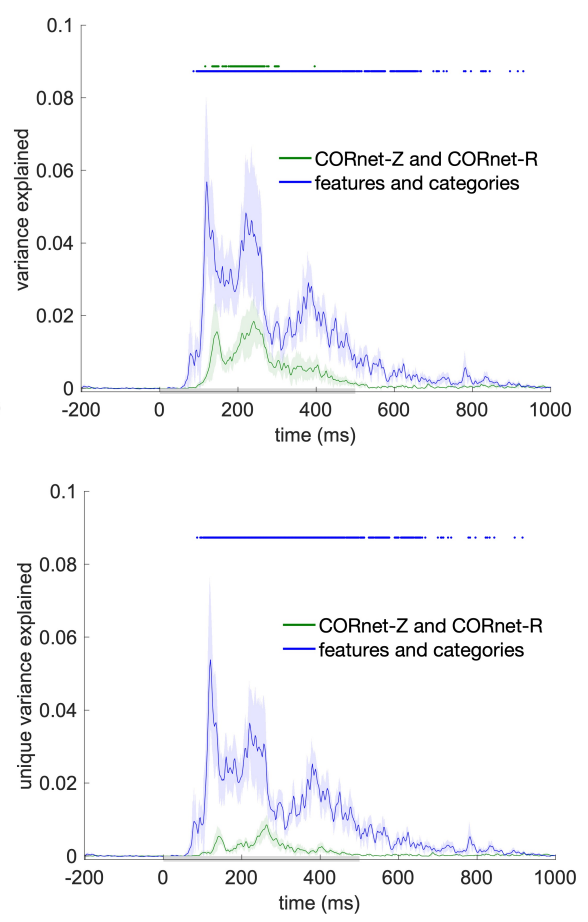

Fig. 2. DNNs better explain lower-level visual representations, visuo-semantic models better explain higher-level visual representations.

a) Variance explained by the visuo-semantic (blue) and DNN (green) models in the source-reconstructed MEG data. To estimate the variance explained by each model class, we took the model components (visuo-semantic features or DNN layers) and ran a non-negative least squares regression. Variance explained was computed as the variance explained by the model predictions in data for images left out during fitting. Significant variance explained is indicated by a horizontal line above the graph (one-sided Wilcoxon signed-rank test, $p<0.05$ corrected). The shaded area around the lines shows the standard error of the mean across participants. The $x$ axis shows time relative to stimulus onset. The gray horizontal bar on the $x$ axis indicates the stimulus duration. b) Unique variance explained by the visuo-semantic and DNN models in the source-reconstructed MEG data. To estimate the unique variance explained by each model class, we took the model predictions (one RDM movie per model class, also used in panel a) and ran a second-level non-negative least squares regression. Unique variance explained was computed by subtracting the variance explained by the reduced GLM (excluding the model class of interest) from the total variance explained by the full GLM (including both model classes). Conventions are the same as in panel a. 
a

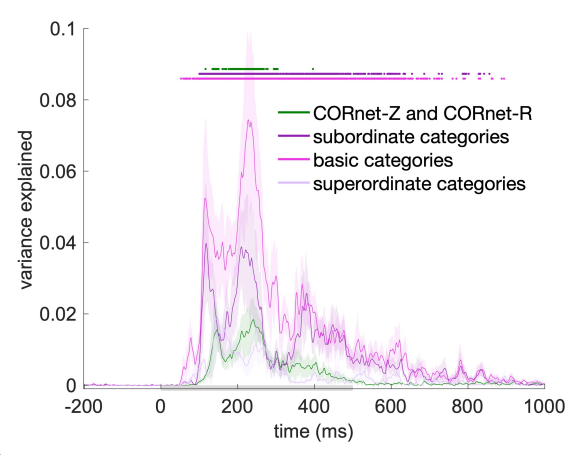

b

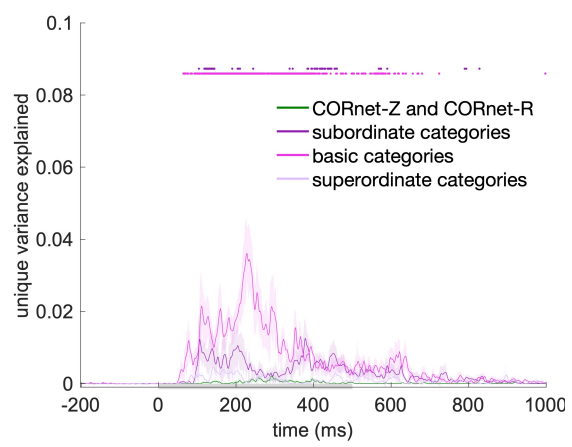

IT/PHC
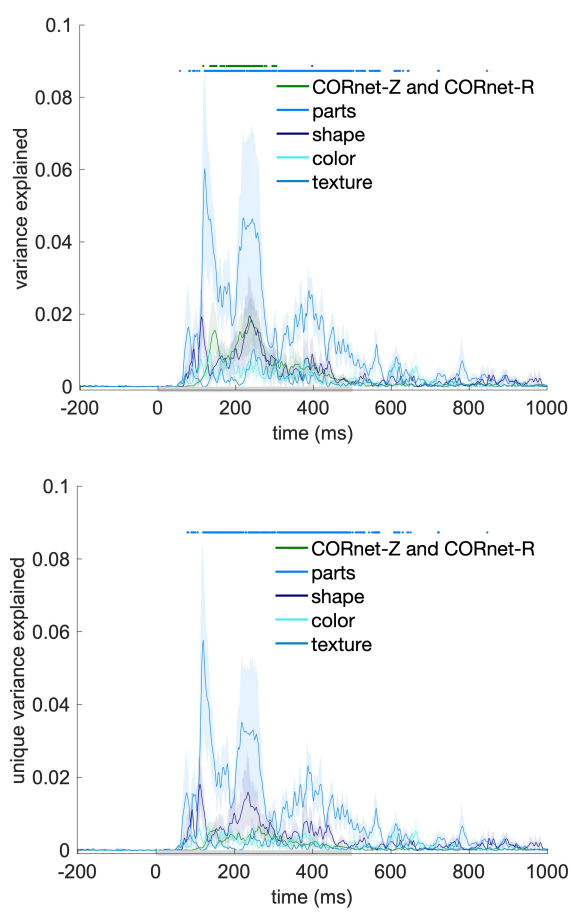
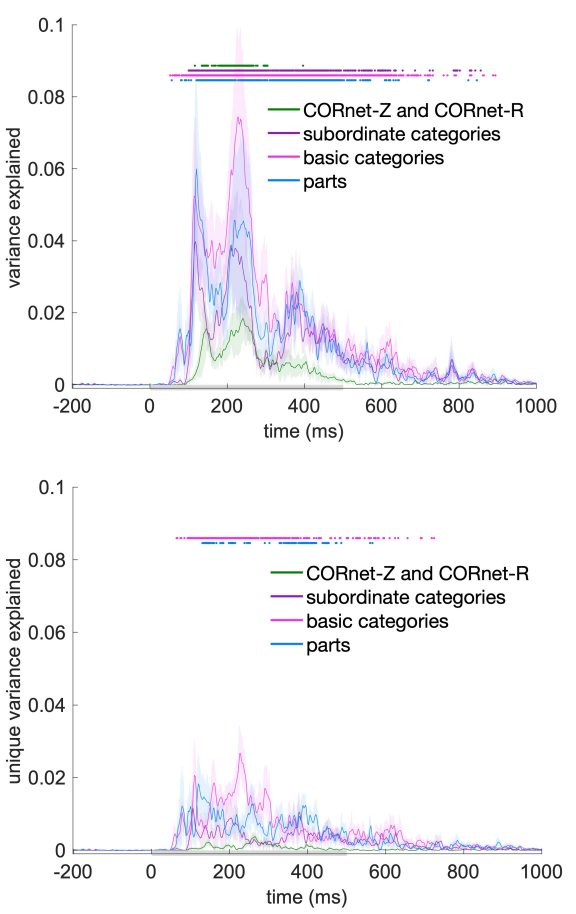

Fig. 3. Object parts and basic categories contribute to the unique variance explained by visuo-semantic models. a) Variance explained by the object features (parts, shape, color, texture; shades of blue), categories (subordinate, basic, superordinate; shades of pink), and DNN model (green) in the source-reconstructed MEG data. Conventions are the same as in Figure 2a. b) Unique variance explained by the features, categories, and DNN model in the source-reconstructed MEG data. Conventions are the same as in Figure 2b.

of unique variance in lower-level and intermediate visual cortex compared to the baseline period. However, for both regions of interest (ROIs), the DNN model explained more unique variance than the visuo-semantic model during the early phase of the response (62 - $128 \mathrm{~ms}$ and 68 - $90 \mathrm{~ms}$ for lower-level and intermediate visual cortex, respectively). For higher-level visual cortex (IT/PHC), only the visuo-semantic model explained unique variance (104 to $640 \mathrm{~ms}$ after stimulus onset, Figure $2 \mathrm{~b}$ ). These results indicate that, in the context of a visuo-semantic predictor, the tested DNNs explain unique variance at lower-level and intermediate, but not higher-level stages of visual processing which instead show a unique contribution of visuo-semantic models. Visuo-semantic models appear to explain components of the higher-level visual representations that DNNs fail to fully capture.

Object parts and basic categories contribute to the unique variance explained by visuo-semantic models. To better understand which components of the visuo-semantic model contribute to explaining unique variance in the higher-level visual representations, we repeated our analyses separately for subsets of object features and subsets of categories. We grouped the visuo-semantic labels into the following subsets: object parts, color, shape, and texture, and subordinate, basic, and superordinate categories (Figure 1b). We found that, among the categories, subordinate and basic categories explained variance in higher-level visual cortex (IT/PHC) (Figure 3a). Furthermore, each of these models explained unique variance in higher-level visual cortex, while the DNN model did not (Figure 3b). Among the object features, only object parts explained variance in higher-level visual cortex (Figure 3a). Furthermore, object parts explained unique variance in higher-level visual cortex, while the DNN model did not (Figure 3b). We next evaluated the three best predictors among the object features and categories together in the context of the DNN predictor. While object parts, subordinate categories, basic categories, and DNNs all explained variance in higher-level visual cortex, only object parts and basic categories explained unique variance (Figure $3 b)$. These results provide clues about which components of the higher-level visual representations DNNs fail to fully capture: object parts and basic categories.

\section{Discussion}

Neural representations of visual objects dynamically unfold over time as we are making sense of the visual world around us. These representational dynamics are thought to reflect the cortical computations that support human object recognition. Here we show that DNNs and human-derived visuo-semantic models explain complementary components of representational dynamics in the human ventral visual stream, estimated via source-reconstructed MEG data. We report a gradual reversal in the importance of DNN and visuo-semantic features from lower- to higher-level visual areas. DNN features explain variance 
over and above visuo-semantic features in lower-level and intermediate visual areas especially during the early phase of the response. In contrast, visuo-semantic features explain variance over and above DNN features in higher-level visual areas during a more prolonged time window. Among the visuo-semantic features, object parts and basic categories drive the explanatory advantage over DNNs. Consistent with our hypothesis, our findings suggest that current DNNs fail to fully capture the visuo-semantic features represented in higher-level human visual cortex, and suggest a path towards more accurate models of ventral stream computations.

Our finding that DNNs outperform visuo-semantic models at explaining lower-level cortical dynamics replicates and extends prior functional magnetic resonance imaging (fMRI) work, which showed that DNNs explain response variance across all stages of the ventral stream while visuo-semantic models predominantly explain response variance in higher-level visual cortex $(14,15,38,42)$. Using source-reconstructed MEG data, we show that the advantage of DNNs over visuo-semantic models is strongest within the first $125 \mathrm{~ms}$ after stimulus onset. During this early time window, the response is likely dominated by feedforward and local recurrent processing as opposed to top-down feedback signals from higher-level areas $(7,12)$. Our results show the importance of analyzing temporally resolved neuroimaging data for revealing when in time competing models account for the rapid dynamic unfolding of human ventral-stream representations. Outcomes can provide clues about the relative importance of these models in accounting for feedforward, locally recurrent, and feedback processes, which is key to understanding the underlying computations of visual inference.

Our findings show that DNNs, despite reaching human-level performance on large-scale object recognition tasks (17), fail to fully capture the visuo-semantic features represented in higher-level human visual cortex, in particular object parts and basic categories. These results suggest that current DNNs fail to fully develop the representational features that give rise to the categorical divisions observed in higher-level visual representations $(29-37,39,40)$. In line with this, prior fMRI work showed that DNNs only adequately accounted for higher-level visual representations after adding new representational features $(14,18,43)$. The new features were created by linearly combining DNN features to emphasize categorical divisions observed in the higher-level visual representations, including the division between faces and nonfaces and between animate and inanimate objects $(14,43)$. Visuo-semantic features, in particular categories, also outperform DNNs at explaining human object similarity judgments (41). DNNs may, at least in part, use different object features for object recognition than humans do. This conclusion is consistent with prior reports that DNNs rely more strongly on lower-level image features such as texture for object categorization (44).

To build more accurate models of human ventral stream computations, we need to provide DNNs with a more human-like learning experience. Two important areas for improvement are visual diet and learning objectives. Each of these shape the internal object representations that develop during visual learning. Humans have a rich visual diet and learn to distinguish between ecologically relevant categories at multiple levels of abstraction, including faces, humans, and animals $(40,45)$. DNNs have a more constrained visual diet and are trained on category divisions that do not entirely match the ones that humans learn in the real world. For example, the most common large-scale image dataset for training DNNs with category supervision (14-18, 22, 28, 43, 46), the ILSVRC 2012 dataset (46), contains subordinate categories that most humans would not be able to distinguish, including dog breeds such as "schipperke" and "groenendael", and lacks some higher-level categories relevant to humans, including "face" and "animal". The path forward is unfolding along two main directions. The first is enrichment of the visual diet of DNNs by better matching the visual variability present in the real world, for example by increasing variability in viewpoint or by training on videos instead of static images $(47,48)$. The second is to more closely match human learning objectives, for example by introducing more human-like category objectives or unsupervised objectives (49-52). Training DNNs on more human-like visual diets and learning objectives may give rise to representational features that more closely match the visuo-semantic features represented in human higher-level visual cortex.

\section{Methods}

Stimuli. Stimuli were 92 colored images of real-world objects spanning a range of categories, including humans, non-human animals, natural objects, and manmade objects (12 human body parts, 12 human faces, 12 animal bodies, 12 animal faces, 23 natural objects, and 21 manmade objects). Objects were segmented from their backgrounds (Figure 1a) and presented to human participants and models on a gray background.

Visuo-semantic models. Feature and category visuo-semantic models have been described in $(40,41)$, where further details can be found.

Definition of visuo-semantic models. To create visuo-semantic models, human observers generated feature labels (e.g., "eye") and category labels (e.g., "animal") for the 92 images (40). The visuo-semantic models are schematically represented in 
Figure $1 \mathrm{~b}$. Feature labels were divided into object parts, colors, textures and shapes, while category labels were divided into subordinate categories, basic categories and superordinate categories. Labels were obtained in a set of two experiments. In Experiment 1, a group of 15 human observers (mean age $=26$ years; 11 females) generated feature and category labels for the object images. In the instruction, we defined features as "visible elements of the shown object, including object parts, colors, textures and shapes". We defined a category as "a group of objects that the shown object is an example of". Observers generated 212 feature labels and 197 category labels. These labels are the model dimensions. In Experiment 2, a separate group of 14 human observers (mean age $=28$ years; seven females) judged the applicability of each model dimension to each image, thereby validating the dimensions generated in Experiment 1, and providing, for each image, its value (present or absent) on each of the dimensions. The image values on the validated model dimensions define the model. To increase the stability of the models during subsequent fitting, highly correlated model dimensions were merged by averaging the image values across these dimensions. The final full feature and category models consisted of 119 and 110 dimensions, respectively.

Construction of the visuo-semantic representational dissimilarity matrices. For each model dimension, we computed, for each pair of images, the squared difference between their values on that dimension. The squared difference reflects the dissimilarity between the two images in a pair. Given that a specific feature or category can either be present or absent in a particular image, image dissimilarities along a single model dimension are binary: they are zero if a feature or category is present or absent in both images, and one if a feature or category is present in one image but absent in the other. The dissimilarities were stored in an RDM, yielding as many RDMs as model dimensions. The full feature RDM model consists of 119 RDMs; the full category RDM model consists of 110 RDMs. Subsets of the feature and category RDM models consist of the following number of RDMs: object parts (82), shape (15), color (10), texture (12), subordinate categories (38), basic categories (67), superordinate categories (5).

Deep neural networks. CORnet-Z and CORnet-R architectures have been described in (22), where further details can be found.

Architecture and training. We used feedforward (CORnet-Z) and locally recurrent (CORnet-R) (22) models in our analyses. The architectures of the two DNNs are schematically represented in Figure 1b. The architecture of CORnets is inspired by the anatomy of monkey visual cortex where each processing stage in the model is thought to correspond to areas V1, V2, V4, and IT respectively (22). The output of the last model area is mapped to the model's behavioral choices using a linear decoder. We chose these DNNs because they have similar architectures with one being feedforward and the other locally recurrent, their architecture is inspired by the primate visual system, they are one of the best models for predicting visual responses in human IT $(27,28)$ and monkey IT $(17)$, and their architectures are relatively simple compared to other DNNs. Each "visual area" in CORnet-Z ("Zero") consists of a single convolution, followed by a ReLU nonlinearity and max pooling. CORnet-R ("Recurrent") introduces local recurrent dynamics within an area. The recurrence occurs only within an area; there are no bypass or feedback connections between areas. For each area, the input is down-scaled twofold and the number of channels is increased twofold by passing the input through a convolution, followed by group normalization (53) and a ReLU nonlinearity. The area's internal state (initially zero) is added to the result and passed through another convolution, again followed by group normalization and a ReLU nonlinearity, resulting in the new internal state of the area. At time step 0 "t0" there is no input to "V2" and above layers, and as a consequence no image-elicited activity is present. From time step "t1" onwards, the imageelicited activity is present in all "visual areas" as the output of the previous area is immediately propagated forward. CORnet-R was trained using five time steps ("t0" - "t4"). Both DNNs were trained on 1.2 million images from the ILSVRC data base (46). The task was to classify each image as containing an object in one of 1,000 possible categories.

Construction of the DNN representational dissimilarity matrices. Representations of the 92 images were computed from the layers of CORnet-Z and CORnet-R. For CORnet-Z, we included the decoder layer and the final processing stage (output) from each "visual area" layer, which resulted in 5 layers. For CORnet-R, we included the decoder layer and the final processing stage from each "visual area" layer for each time step, which resulted in 21 layers. For each layer of CORnet-Z and CORnet-R, we extracted the unit activations in response to the images and converted these into one activation vector per image. For each pair of images, we computed the dissimilarity ( 1 minus Spearman's correlation) between the activation vectors. This yielded an RDM for each DNN layer. The resulting RDMs capture which stimulus information is emphasized and which is de-emphasized by the DNNs at different stages of processing.

MEG source-reconstructed data. Acquisition and analysis of the MEG data have been described in (6), where further details can be found. The source reconstruction of the MEG data has been described in (10).

Participants. Sixteen healthy human volunteers participated in the MEG experiment (mean age $=26,10$ females). MEG source reconstruction analyses were performed for a subset of 15 participants for whom structural and functional MRI data were acquired. Participants had normal or corrected-to-normal vision. Before scanning, the participants received information about the procedure of the experiment and gave their written informed consent for participating. The experiment was conducted 
in accordance with the Ethics Committee of the Massachusetts Institute of Technology Institutional Review Board and the Declaration of Helsinki.

Experimental design and task. Stimuli were presented at the centre of the screen for $500 \mathrm{~ms}$, while participants performed a paper clip detection task. Stimuli were overlaid with a light gray fixation cross and displayed at a width of $2.9^{\circ}$ visual angle. Participants completed 10 to 14 runs. Each image was presented twice in every run in random order. Participants were asked to press a button and blink their eyes in response to a paper clip image shown randomly every 3 to 5 trials. These trials were excluded from further analyses. Each participant completed two MEG sessions.

MEG data acquisition and preprocessing. MEG signals were acquired from 306 channels (204 planar gradiometers, 102 magnetometers) using an Elekta Neuromag TRIUX system (Elekta) at a sampling rate of 1,000 Hz. The data were bandpass filtered between 0.03 and $330 \mathrm{~Hz}$, cleaned using spatiotemporal filtering, and down-sampled to $500 \mathrm{~Hz}$. Baseline correction was performed using a time window of $100 \mathrm{~ms}$ before stimulus onset.

MEG source reconstruction. The source reconstructions were performed using the MNE Python toolbox (54). We used participant individual structural $\mathrm{T} 1$ scans to obtain volume conduction estimates using single layer boundary element models based on the inner skull boundary. The source space consisted of 10,242 source points per hemisphere, positioned along the gray/white matter boundary estimated via FreeSurfer. We defined source orientations as surface normals with a loose orientation constraint. We used an iterative procedure for MEG/MRI alignment based on fiducials and digitizer points along the head surface. We estimated the sensor noise covariance matrix from the baseline period ( $100 \mathrm{~ms}$ to $0 \mathrm{~ms}$ before stimulus onset) and regularized it according to the Ledoit-Wolf procedure (55). We projected source activations onto the surface normal, obtaining one activation estimate per point in source space and time.

Definition of regions of interest. We used a multimodal brain atlas (56) to define ROIs. We defined three ROIs covering lowlevel (V1-3), intermediate (V4t/LO1-3), and high-level visual areas (IT/PHC, consisting of TE1-2p, FFC, VVC, VMV2-3, PHA1-3). We converted the atlas annotation files to fsaverage coordinates (57) and mapped them to each participant using spherical averaging.

Construction of the MEG representational dissimilarity matrices. We computed temporally changing RDM movies from the source-reconstructed MEG data for each participant, ROI, hemisphere, and session. We first extracted a trial-average multivariate source time series for each stimulus. We then computed an RDM at each time point by estimating the pattern distance between all pairs of images using correlation distance (1 minus Pearson correlation). The RDM movies were averaged across hemispheres and sessions, resulting in one RDM movie for each participant and ROI.

Weighted representational modeling. Weighted representational modeling used here has been described in $(14,40,41,43$, 58). To improve model performance, we linearly combined the different model dimensions (features and categories for the visuo-semantic models, layers for the DNNs) to yield an object representation that best predicts the source-reconstructed MEG data. We estimated the representational weights, one for each single-dimension RDM, using regularized (L2) linear regression, implemented in MATLAB using glmnet. Glmnet implements elastic net regularized regression using cyclical coordinate descent. We used standard settings (including standardization of the predictors before fitting), except that we constrained the weights to be non-negative. To prevent biased model performance estimates due to overfitting to a particular set of images, model performance was estimated by cross-validation to a subset of the images held out during model fitting. For each crossvalidation fold, we randomly selected 84 of the 92 images as the training set and used the corresponding pairwise dissimilarities to estimate the model weights. The model weights were then used to predict the pairwise dissimilarities for the eight left-out images. This procedure was repeated until predictions were obtained for all pairwise dissimilarities. For each cross-validation fold, we determined the best regularization parameter (i.e. the one with the minimum squared error between prediction and data) using nested cross-validation to held-out images within the training set. We performed the fitting procedure for each participant and for each time point of the MEG source-reconstructed data.

Variance and unique variance analysis. We used a GLM to evaluate variance and unique variance explained by the models in the source-reconstructed MEG data (10). For each model $\mathrm{m}$, the variance explained $R^{2}$ was computed by GLM fitting using $\mathrm{X}=$ "model $\mathrm{m}$ " and $\mathrm{Y}=$ data. For each model $\mathrm{m}$, the unique variance explained was computed by subtracting the total variance explained by the reduced GLM (excluding the model of interest) from the total variance explained by the full GLM. Specifically, for model $\mathrm{m}$, we fit the GLM using $\mathrm{X}=$ "all models but $\mathrm{m}$ " and $\mathrm{Y}=$ data, then we subtracted the resulting $R^{2}$ from the total $R^{2}$ (fit the GLM using $\mathrm{X}=$ "all models" and $\mathrm{Y}=$ data). We performed this procedure for each participant and ROI. We used non-negative least squares to find optimal weights as RDMs consist of dissimilarity estimates, which cannot be negative. A constant term was included in the GLM model to correct for homogeneous changes in dissimilarity across the whole RDM. To evaluate the significance of the variance explained and unique variance explained contributed by each model across participants, we performed a one-sided Wilcoxon signed-rank test, controlling the expected false discovery rate at 0.05 . 
bioRxiv preprint doi: https://doi.org/10.1101/2021.10.25.465583; this version posted October 26, 2021. The copyright holder for this preprint (which was not certified by peer review) is the author/funder, who has granted bioRxiv a license to display the preprint in perpetuity. It is made available under aCC-BY-NC-ND 4.0 International license.

Variance and unique variance explained and standard error lines were low-pass filtered at $80 \mathrm{~Hz}$ for easier visibility. Statistical inference is based on the unsmoothed data.

\section{ACKNOWLEDGEMENTS}

This research was supported by the Wellcome Trust (grant number 206521/Z/17/Z) awarded to KMJ; the Alexander von Humboldt Foundation postdoctoral fellowship awarded to KMJ, the German Research Council grants (Cl241/1-1, Cl241/3-1 Cl241/7-1) awarded to RMC, the European Research Council grant (ERC-StG-2018-803370) awarded to RMC, and an NSERC Discovery Grant (RGPIN-2019-06741) awarded to MM. We thank Martin Schrimpf for giving his input on the CORnets Methods section. For the purpose of open access, the author has applied a CC BY public copyright licence to any Author Accepted Manuscript version arising from this submission.

\section{COMPETING FINANCIAL INTERESTS}

The authors declare no competing interests.

\section{AUTHOR CONTRIBUTIONS}

KMJ, TCK, RMC, NK and MM designed the experiments. KMJ collected behavioral data for visuo-semantic model building. RMC collected MEG data. TCK provided sourcereconstructed MEG data and second-level GLM code. KMJ performed the analyses. KMJ wrote the paper. All authors edited the paper. NK and MM supervised the work.

\section{DATA AVAILABILITY}

The datasets generated during the current study are available from the corresponding authors on reasonable request.

\section{CODE AVAILABILITY}

The code generated during the current study is available from the corresponding authors on reasonable request.

\section{References}

1. Yasuko Sugase, Shigeru Yamane, Shoogo Ueno, and Kenji Kawano. Global and fine information coded by single neurons in the temporal visual cortex. Nature, 400(6747):869-873, 1999.

2. Chou P. Hung, Gabriel Kreiman, Tomaso Poggio, and James J. DiCarlo. Fast Readout of Object Identity from Macaque Inferior Temporal Cortex. Science, 310(5749):863-866, 2005.

3. Ethan M. Meyers, David J. Freedman, Gabriel Kreiman, Earl K. Miller, and Tomaso Poggio. Dynamic Population Coding of Category Information in Inferior Temporal and Prefrontal Cortex. Journal of Neurophysiology, 100(3):1407-1419, 2008.

4. Thomas Carlson, David A Tovar, and Nikolaus Kriegeskorte. Representational dynamics of object vision : The first 1000 ms. Journal of Vision, 13:1-19, 2013.

5. Alex Clarke, Kirsten I. Taylor, Barry Devereux, Billi Randall, and Lorraine K. Tyler. From Perception to Conception: How Meaningful Objects Are Processed over Time. Cerebral Cortex, 23(1): 187-197, 2013.

6. Radoslaw Martin Cichy, Dimitrios Pantazis, and Aude Oliva. Resolving human object recognition in space and time. Nature Neuroscience, 17(3):455-62, 2014

7. Leyla Isik, Ethan M. Meyers, Joel Z. Leibo, and Tomaso Poggio. The dynamics of invariant object recognition in the human visual system. Journal of Neurophysiology, 111(1):91-102, 2014.

8. Avniel Singh Ghuman, Nicolas M. Brunet, Yuanning Li, Roma O. Konecky, John A. Pyles, Shawn A. Walls, Vincent Destefino, Wei Wang, and R. Mark Richardson. Dynamic encoding of face information in the human fusiform gyrus. Nature Communications, 5(1):5672, 2014

9. Martin N Hebart, Brett B Bankson, Assaf Harel, Chris I Baker, and Radoslaw M Cichy. The representational dynamics of task and object processing in humans. eLife, 7:e32816, 2018.

10. Tim C. Kietzmann, Courtney J. Spoerer, Lynn K. A. Sörensen, Radoslaw M. Cichy, Olaf Hauk, and Nikolaus Kriegeskorte. Recurrence required to capture the dynamic computations of the human ventral visual stream. Proceedings of the National Academy of Sciences, (43):21854-21863, 2019.

11. Philippe Beaudoin Joel Zylberberg Denis Therien Blake A. Richards, Timothy P. Lillicrap and Konrad P. Kording. A deep learning framework for neuroscience. Nature Neuroscience, 22:10, 2019.

12. Tim C. Kietzmann, Patrick McClure, and Nikolaus Kriegeskorte. Deep Neural Networks in Computational Neuroscience. Oxford University Press, 2019.

13. Daniel L K Yamins, Ha Hong, Charles F Cadieu, Ethan a Solomon, Darren Seibert, and James J DiCarlo. Performance-optimized hierarchical models predict neural responses in higher visual cortex. Proceedings of the National Academy of Sciences of the United States of America, 111:8619-24, 2014.

14. Seyed-Mahdi Khaligh-Razavi and Nikolaus Kriegeskorte. Deep Supervised, but Not Unsupervised, Models May Explain IT Cortical Representation. PLoS Computational Biology, 10(11):e1003915, 2014.

15. U. Güçlü and M. a. J. van Gerven. Deep Neural Networks Reveal a Gradient in the Complexity of Neural Representations across the Ventral Stream. Journal of Neuroscience, 35(27):10005-10014 2015.

16. Radoslaw Martin Cichy, Aditya Khosla, Dimitrios Pantazis, and Antonio Torralba. Comparison of deep neural networks to spatio-temporal cortical dynamics of human visual object recognition reveals hierarchical correspondence. Scientific Reports, 2017.

17. Martin Schrimpf, Jonas Kubilius, Ha Hong, Elias B Issa, Kohitij Kar, Jonathan Prescott-Roy, Rishi Rajalingham, Daniel L K Yamins, and James J DiCarlo. Brain-Score: Which Artificial Neural Network is most Brain-Like? bioRxiv, 2018.

18. Katherine R Storrs, Tim C Kietzmann, Alexander Walther, Johannes Mehrer, and Nikolaus Kriegeskorte. Diverse deep neural networks all predict human it well, after training and fitting. Journal of Cognitive Neuroscience, 33:2044-2064, 2020.

19. Randall C. O'Reilly, Dean Wyatte, Seth Herd, Brian Mingus, and David J. Jilk. Recurrent processing during object recognition. Frontiers in Psychology, 4(APR):1-14, 2013.

20. Qianli Liao and Tomaso Poggio. Bridging the Gaps Between Residual Learning, Recurrent Neural Networks and Visual Cortex. (047):1-16, 2016.

21. Courtney J. Spoerer, Patrick McClure, and Nikolaus Kriegeskorte. Recurrent Convolutional Neural Networks: A Better Model of Biological Object Recognition. Frontiers in Psychology, 8:1551, 2017.

22. Jonas Kubilius, Martin Schrimpf, Aran Nayebi, Daniel Bear, Daniel L. K. Yamins, and James J. DiCarlo. CORnet: Modeling the Neural Mechanisms of Core Object Recognition. 2018.

23. Hanlin Tang, Martin Schrimpf, William Lotter, Charlotte Moerman, Ana Paredes, Josue Ortega Caro, Walter Hardesty, David Cox, and Gabriel Kreiman. Recurrent computations for visual pattern completion. Proceedings of the National Academy of Sciences, page 201719397, 2018.

24. Kohitij Kar, Jonas Kubilius, Kailyn Schmidt, Elias B. Issa, and James J. DiCarlo. Evidence that recurrent circuits are critical to the ventral stream's execution of core object recognition behavior. Nature Neuroscience, 22(6):974-983, 2019.

25. Karim Rajaei, Yalda Mohsenzadeh, Reza Ebrahimpour, and Seyed-Mahdi Khaligh-Razavi. Beyond core object recognition: Recurrent processes account for object recognition under occlusion. PLoS Computational Biology, page 30, 2019.

26. Courtney J. Spoerer, Tim C. Kietzmann, Johannes Mehrer, lan Charest, and Nikolaus Kriegeskorte. Recurrent neural networks can explain flexible trading of speed and accuracy in biological vision. PLOS Computational Biology, 16(10):e1008215, 2020.

27. Kamila Maria Jozwik, Martin Schrimpf, Nancy Kanwisher, and James J. DiCarlo. To find better neural network models of human vision, find better neural network models of primate vision. bioRxiv, 2019.

28. Kamila M Jozwik, Michael Lee, Tiago Marques, Martin Schrimpf, and Pouya Bashivan. Large-scale hyperparameter search for predicting human brain responses in the Algonauts challenge. bioRxiv, 2019.

29. K Tanaka. Inferotemporal cortex and object vision. Annual Review of Neuroscience, 19:109-139, 1996.

30. Yukako Yamane, Eric T Carlson, Katherine C Bowman, Zhihong Wang, and Charles E Connor. A neural code for three-dimensional object shape in macaque inferotemporal cortex. Nature Neuroscience, 11(11):1352-1360, 2008.

31. Winrich A Freiwald, Doris Y Tsao, and Margaret S Livingstone. A face feature space in the macaque temporal lobe. Nature Neuroscience, 12(9):1187-1196, 2009.

32. Elias B Issa and James J DiCarlo. Precedence of the eye region in neural processing of faces. The Journal of Neuroscience, 32(47):16666-82, 2012.

33. N Kanwisher, J McDermott, and M M Chun. The fusiform face area: a module in human extrastriate cortex specialized for face perception. The Journal of Neuroscience, 17(11):4302-4311, 1997.

34. R Epstein and N Kanwisher. A cortical representation of the local visual environment. Nature, 392(April):598-601, 1998.

35. Paul E Downing, Yuhong Jiang, Miles Shuman, and Nancy Kanwisher. A Cortical Area Selective for Visual Processing of the Human Body. Science, 293:2470-2473, 2001.

36. J V Haxby, M I Gobbini, M L Furey, a Ishai, J L Schouten, and P Pietrini. Distributed and overlapping representations of faces and objects in ventral temporal cortex. Science, 293(5539):2425-2430, 2001.

37. Nikolaus Kriegeskorte, Marieke Mur, Douglas a. Ruff, Roozbeh Kiani, Jerzy Bodurka, Hossein Esteky, Keiji Tanaka, and Peter a. Bandettini. Matching Categorical Object Representations in Inferior Temporal Cortex of Man and Monkey. Neuron, 60(6):1126-1141, 2008.

38. Alexander G. Huth, Shinji Nishimoto, An T. Vu, and Jack L. Gallant. A Continuous Semantic Space Describes the Representation of Thousands of Object and Action Categories across the Human Brain. Neuron, 76(6):1210-1224, 2012. 
bioRxiv preprint doi: https://doi.org/10.1101/2021.10.25.465583; this version posted October 26, 2021. The copyright holder for this preprint (which was not certified by peer review) is the author/funder, who has granted bioRxiv a license to display the preprint in perpetuity. It is made available under aCC-BY-NC-ND 4.0 International license.

39. M. Mur, D. a. Ruff, J. Bodurka, P. De Weerd, P. a. Bandettini, and N. Kriegeskorte. Categorical, Yet Graded - Single-Image Activation Profiles of Human Category-Selective Cortical Regions. Journal of Neuroscience, 32(25):8649-8662, 2012.

40. Kamila M. Jozwik, Nikolaus Kriegeskorte, and Marieke Mur. Visual features as stepping stones toward semantics: Explaining object similarity in IT and perception with non-negative least squares. Neuropsychologia, 83:201-226, 2016.

41. Kamila M. Jozwik, Nikolaus Kriegeskorte, Katherine R. Storrs, and Marieke Mur. Deep Convolutional Neural Networks Outperform Feature-Based But Not Categorical Models in Explaining Object Similarity Judgments. Frontiers in Psychology, 8:1726, 2017.

42. Kamila Maria Jozwik, Nikolaus Kriegeskorte, Radoslaw Martin Cichy, and Marieke Mur. Deep convolutional neural networks, features, and categories perform similarly at explaining primate high-level visual representations. Conference on Cognitive Computational Neuroscience, 2018.

43. Katherine R. Storrs, Seyed-Mahdi Khaligh-Razavi, and Nikolaus Kriegeskorte. Noise ceiling on the crossvalidated performance of reweighted models of representational dissimilarity: Addendum to Khaligh-Razavi \& Kriegeskorte (2014). bioRxiv, 2020.

44. Robert Geirhos, Patricia Rubisch, Claudio Michaelis, Matthias Bethge, Felix A. Wichmann, and Wieland Brendel. Imagenet-trained cnns are biased towards texture; increasing shape bias improves accuracy and robustness. arXiv, 2019.

45. Marieke Mur, Mirjam Meys, Jerzy Bodurka, Rainer Goebel, Peter Bandettini, and Nikolaus Kriegeskorte. Human object-similarity judgments reflect and transcend the primate-it object representation. Frontiers in Psychology, 4:128, 2013.

46. Olga Russakovsky, Jia Deng, Hao Su, Jonathan Krause, Sanjeev Satheesh, Sean Ma, Zhiheng Huang, Andrej Karpathy, Aditya Khosla, Michael Bernstein, Alexander C. Berg, and Li Fei-Fei. ImageNet large scale visual recognition challenge. International Journal of Computer Vision, 115:211-252, 2015.

47. Andrei Barbu, David Mayo, Julian Alverio, William Luo, Christopher Wang, Dan Gutfreund, Josh Tenenbaum, and Boris Katz. ObjectNet: A large-scale bias-controlled dataset for pushing the limits of object recognition models. Advances in Neural Information Processing Systems, 2019.

48. Chengxu Zhuang, Alex Andonian, and Daniel Yamins. Unsupervised learning from video with deep neural embeddings. CoRR, abs/1905.11954, 2019.

49. Johannes Mehrer, Courtney J. Spoerer, Emer C. Jones, Nikolaus Kriegeskorte, and Tim C. Kietzmann. An ecologically motivated image dataset for deep learning yields better models of human vision. Proceedings of the National Academy of Sciences, 118(8):e2011417118, 2021.

50. Irina Higgins, Le Chang, Victoria Langston, Demis Hassabis, Christopher Summerfield, Doris Tsao, and Matthew Botvinick. Unsupervised deep learning identifies semantic disentanglement in single inferotemporal neurons. arXiv, 2020.

51. Chengxu Zhuang, Siming Yan, Aran Nayebi, Martin Schrimpf, Michael C. Frank, James J. DiCarlo, and Daniel L. K. Yamins. Unsupervised neural network models of the ventral visual stream. Proceedings of the National Academy of Sciences, 118(3):e2014196118, 2021.

52. Talia Konkle and George A. Alvarez. Instance-level contrastive learning yields human brain-like representation without category-supervision. bioRxiv, 2020.

53. Yuxin Wu and Kaiming He. Group Normalization. arXiv, 2018.

54. Alexandre Gramfort. MEG and EEG data analysis with MNE-Python. Frontiers in Neuroscience, 7, 2013.

55. Olivier Ledoit and Michael Wolf. A well-conditioned estimator for large-dimensional covariance matrices. Journal of Multivariate Analysis, 88(2):365-411, 2004.

56. Matthew F. Glasser, Timothy S. Coalson, Emma C. Robinson, Carl D. Hacker, John Harwell, Essa Yacoub, Kamil Ugurbil, Jesper Andersson, Christian F. Beckmann, Mark Jenkinson, Stephen M. Smith, and David C. Van Essen. A multi-modal parcellation of human cerebral cortex. Nature, 536(7615):171-178, 2016.

57. Bruce Fischl, Martin I Sereno, Roger B H Tootell, and Anders M Dale. High-resolution intersubject averaging and a coordinate system for the cortical surface. Human Brain Mapping, 1999.

58. Philipp Kaniuth and Martin N. Hebart. Feature-reweighted RSA: A method for improving the fit between computational models, brains, and behavior. bioRxiv, 2021. 